\title{
LOS ROLES DE GÉNERO Y SU PAPEL EN LAS ACTITUDES Y COMPORTAMIENTOS AFECTIVO- SEXUALES: UN ESTUDIO SOBRE ADOLESCENTES SALMANTINOS
}

\author{
Lidia Lobato \\ lidialobatoperez@usal.es \\ Universidad de Salamanca - España \\ Manuel Rodríguez-Becerra \\ mrodriguezbe@upsa.es \\ Universidad Pontificia de Salamanca - España
}

\author{
Cristina Jenaro \\ crisje@usal.es \\ Universidad de Salamanca - España \\ Noelia Flores \\ nrobaina@usal.es \\ Universidad de Salamanca - España
}

Recibido: 29-02-2016

Aceptado: 11-05-2016

\section{Resumen}

En el presente estudio centramos nuestro interés en evaluar las actitudes, conocimientos y comportamientos de los adolescentes respecto a la sexualidad y sus prácticas, así como el impacto de variables como el género en estas valoraciones. Metodología. El estudio se ha llevado a cabo con una muestra de conveniencia de 141 estudiantes de Bachillerato o de módulos profesionales residentes en Salamanca, España. Dichos alumnos fueron evaluados mediante el Cuestionario para adolescentes sobre educación sexual. Resultados. Los datos indican que existe un amplio desconocimiento sobre temas básicos relacionados con el uso del preservativo o el embarazo. Se encuentran además claras relaciones entre el género y actitudes hacia la homosexualidad, la prostitución, la consideración de la sexualidad o la propia actividad sexual. Discusión. Es necesario llevar a cabo programas formativos que mejoren los conocimientos y fomenten actitudes y conductas positivas y saludables y respetuosas hacia la sexualidad propia y ajena.

Palabras Clave: Educación sexual y afectiva, conducta sexual, actitud sexual, diferencias de género, educación post-obligatoria, evaluación.

\begin{abstract}
In this study, we focused our interest on assessing the attitudes, knowledge and behaviors of adolescents about sexuality and their practices as well as the impact of variables such as gender on these ratings. Methodology. The study was conducted with a convenience sample of 141 high school or professional modules students from Salamanca, Spain. These students were assessed using the Questionnaire for teens on sex education. Results. The data indicate that there is widespread lack of knowledge about basic issues related to the use of condoms and pregnancy. A clear relationship was also found between gender and attitudes toward homosexuality, prostitution, vision of sexuality and sexual activity itself. Discussion. It is necessary to conduct training programs to improve knowledge and promote positive attitudes and behaviors, that are healthy and respectful towards their own and others sexuality.
\end{abstract}

Keywords: Affective and sexual education, sexual behavior, sexual attitude, gender differences, post-secondary education, evaluation. 


\section{Introducción}

Los centros educativos forman a los alumnos no sólo en conocimientos y competencias, sino también en valores. Son por tanto una vía para interiorizar normas de género y sexualidad, entre otras (Platero Méndez, 2010). Si bien se está avanzando en este terreno, todavía queda mucho por hacer para alejarse de modelos de género más tradicionales (Carrillo, Prat, Guitart, and Fatsini, 2010; da Silva, 2013). Así, como indica González Pérez (2010), en muchos casos tanto los materiales curriculares como las prácticas educativas contribuyen a transmitir mensajes discriminatorios para las mujeres, tratando a unos y otros de forma distinta.

Una de las vías para formar a los estudiantes en valores igualitarios es a través de la educación afectivo sexual. De hecho, desde la implantación de la LOGSE en España (1990), tanto la educación afectivo sexual como la educación para la salud constituyen ejes transversales del currículo y que, en consecuencia, contribuyen a la formación integral del alumnado. Antes de proseguir, deseamos aclarar qué entendemos por 'educación afectivo sexual'. Asumimos para ello los Estándares de Educación Sexual para Europa de la Organización Mundial de la Salud (WHO Regional Office for Europe 2010: 26) que define la educación sexual en un sentido amplio como "el aprendizaje sobre los aspectos cognitivos, emocionales, sociales, relacionales y físicos de la sexualidad [...]. La educación sexual deber servir para proteger y apoyar un desarrollo seguro de la sexualidad en la infancia y juventud. Ha de preparar a niños, niñas y jóvenes a través de información, habilidades y valores positivos para que comprendan, disfruten de su sexualidad y de sus relaciones de manera segura y satisfactoria, responsabilizándose de su propia salud sexual y bienestar y la de otras personas. Debe permitirles que puedan hacer elecciones que mejoren sus vidas y contribuyan a hacer una sociedad más compasiva”.

Asumir esta definición requiere apostar por un abordaje desde una concepción más general y menos reduccionista que lo limitado al acto sexual, anticoncepción o embarazos, en consonancia con las propuestas de otros autores (Sáez Sesma, 2009). Tenemos también en cuenta que, de acuerdo con la revisión realizada por Muñoz Cadavid y Revenga Sánchez (2005), la educación afectivo sexual en las escuelas requiere servirse de diferentes enfoques: por un lado, del aprendizaje de actitudes, lo que supone adquirir no solo respuestas afectivas, cognitivas y conductuales sino también destrezas comunicativas para expresar la experiencia subjetiva ante el hecho sexual y sus diferentes manifestaciones. Por otro lado, un aprendizaje ético regido por principios universales. En tercer lugar, un aprendizaje de la identidad sexual y de género que evite prejuicios y estereotipos, para lo que la escuela y educadores juegan un papel fundamental (Muñoz Cadavid y Revenga Sánchez, 2005). Una concepción de la educación afectivo sexual abierta, democrática, integral y profesional, debe permitir asumirse positivamente como seres sexuados, comprender adecuadamente el hecho sexual humano, cultivar una ética para las relaciones interpersonales y adquirir habilidades para la construcción 
de unas relaciones saludables, satisfactorias, responsables y no discriminatorias por razones de género u orientación sexual (Colectivo Harimaguada, 2007).

Si bien estos principios parecen claros y gozar de un elevado consenso, en la práctica no es todo tan sencillo. En este sentido, como indica Palomino (2003), la introducción en la escuela de la educación afectiva y sexual ha sido siempre polémica. En muchos casos, en las prácticas profesionales de salud y en la tarea docente en los centros educativos se siguen manejando los conceptos biológicos de la sexualidad. Así por ejemplo, las diversas campañas de educación sexual realizadas en España en los últimos 15 años se han dirigido fundamentalmente a la población adolescente y a promocionar el 'sexo seguro', con resultados manifiestamente mejorables (Jara Rascón y Alonso Sandoica, 2008). Al mismo tiempo, en estos sistemas tan importantes se continúan desaprobando conductas consideradas "sexuales" e "inapropiadas" y se siguen valorando negativamente aquellas feminidades y masculinidades que no se ajustan a las imágenes tradicionales de mujer y varón. También existen preconceptos acerca de la autoridad que dificultan el acercamiento a los adolescentes. Con frecuencia, la relación con estos últimos genera en los padres una gran incertidumbre, situándose éstos en posiciones extremas que oscilan desde el patrón autoritario al patrón permisivo. De igual manera, existe la idea generalizada de que la educación se debe basar en un conjunto de saberes técnicos y no en un conjunto de saberes que, si bien no son técnicos, sí son muy valiosos vitalmente. Debido a esto, la afectividad y la sexualidad se han visto excluidas, por norma general, del proceso pedagógico.

La educación, como transmisora de los valores, costumbres creencias y patrones de comportamiento de una sociedad, también se postula como una de las principales vías de transmisión de género. Asimismo, la selección de conocimientos relacionados con la sexualidad y la afectividad está determinada por las connotaciones ideológicas y religiosas de una estructura determinada, mayoritariamente patriarcal, que dificultan en ocasiones, el establecimiento de una buena educación afectiva y sexual en el contexto educativo (Abad, 2002).

La revisión de estudios sobre el tema pone de manifiesto que un gran número de programas de intervención en la escuela, se centra en la prevención de las enfermedades de transmisión sexual (ETS), particularmente del VIH/SIDA, así como de los embarazos (Anabitarte Rivas, 2002; Bataller i Perelló, 2002; Espada Sánchez y Quiles Sebastián, 2002; García Martínez, 2005; Igartúa Perosanz y Rodríguez Bravo, 2002; Planes Pedra et al., 2004; Ubillos Landa y Mayordomo López, 2003; Usieto y Sastre, 2002). Estos y otros programas parten de la evidencia de que la juventud es un grupo de riesgo por sus prácticas sexuales, por lo que es necesaria una educación sexual desde edades tempranas (Diz, Sueiro, Chas y Diéguez, 2003).

En nuestra opinión, dicha educación sexual debe ir más allá de los aspectos asociados al sexo, esto es, a las características anatómicas y fisiológicas de los organismos vivientes que pueden ser identificadas en los niveles cromosómico, génico, hormonal y genital (Ortíz, 2004). Así, es fundamental que aborde un tema tan sustantivo como el género. Dicho género es el significado que las sociedades atribuyen a los rasgos biológicos asociados con el sexo; se trata de construcciones históricas, culturales y arbitrarias. Si bien se distinguen dos géneros básicos: 
masculino y femenino, en la realidad existe un amplio espectro entre estos dos modelos e incluso fuera de estas dos categoría, como son las personas andróginas (Caricote Agreda, 2006; Ortiz y Granados, 2003).

Este significado o sistema de géneros puede dar lugar a estereotipos de género esto es, a un conjunto de creencias, prescripciones, reglas, expectativas y atribuciones - la mayoría de las veces implícitas - de cómo deben ser hombres y mujeres. Dichos estereotipos definen el "deber ser" en función del sexo. Además, se puede relacionar con otras ideologías como el androcentrismo superioridad de lo masculino frente a lo femenino- y el heterosexismo - es el sistema ideológico que niega, denigra y estigmatiza cualquier forma de conducta, identidad o relación diferentes de las heterosexuales (Costa, 2006; Ortiz, 2004). Como afirma González Gabaldón (1999), la relación entre los conceptos estereotipo, prejuicio y discriminación es muy estrecha.

En esta línea, también se deben abordar contenidos como la identidad de género - experiencia subjetiva de pertenencia a uno de los géneros- y el rol de género que comprende conductas, vestimenta, expresiones corporales, posturas, presencia, porte y gestos (Corona, 1994; Ortiz, 2004).

Así pues, el género, los roles y estereotipos asociados al mismo y su relación con los aspectos afectivos y sexuales hace necesario abordar otros temas en una educación comprensiva de estos contenidos. El abordaje de los aspectos antes comentados se relaciona estrechamente y permite entender y contextualizar fenómenos como la violencia de género. La ley Orgánica 1/2004, del 28 de diciembre, de Medidas de Protección Integral contra la Violencia de Género, aprobada en España define la violencia de género como aquella que "como manifestación de la discriminación, la situación de desigualdad y las relaciones de poder de los hombres sobre las mujeres, se ejerce sobre éstas por parte de quienes sean o hayan sido sus cónyuges o de quienes estén o hayan estado ligados a ellas por relaciones similares de afectividad, aun sin convivencia", y "comprende todo acto de violencia física y psicológica, incluidas las agresiones a la libertad sexual, las amenazas, las coacciones o la privación arbitraria de libertad" (art. 1).

Como la propia definición pone de manifiesto, en esta educación deben tener también cabida los delitos contra la libertad sexual, dentro de la cual se encuentra la prostitución. La regulación, de la prostitución en España, viene recogida en la Ley Orgánica 10/1995, de 23 de noviembre, por la que se aprueba el Código Penal. En éste, no se penaliza a quien la ejerce pero sí la explotación derivada de dicho ejercicio cuando medien determinados factores (empleo de violencia, intimidación o engaño, o abuso de una situación de superioridad o de necesidad o vulnerabilidad de la víctima, edad, etc.). Continuando con el tema de la violencia de género, un aspecto que crea creciente preocupación es la violencia en parejas jóvenes. Así, la experiencia de violencia en el noviazgo se produce con una frecuencia considerable en parejas adolescentes, siendo más comunes las de tipo sexual y psicológico (Samaniego García y Freixas Farré, 2010). De ahí que se hayan elaborado programas para intervenir en este sentido (Hernando Gómez, 2007; Pérez Jiménez, 2010).

En respuesta a estas realidades sociales cada vez más visibles, los programas de educación afectivo sexual cada vez más están incluyen contenidos al respecto. Por ejemplo, algunos 
acentúan aspectos relacionados con el autoconcepto, autoestima, o la ideología del rol sexual (Carrera-Fernández et al., 2007), o directamente abordan el tema de la violencia de género (Colectivo Harimaguada, 2007). El abordaje de estos temas es ineludible, a tenor de la asociación entre actitudes hacia la sexualidad y conductas sexuales (Sueiro Domínguez, Diéguez, Chas, y Diz, 2004), actitudes sexistas y violencia de género (González Pozuelo, 2008) o entre la violencia, el matonismo y otras formas de violencia de género, incluyendo la homofobia, el acoso sexual, el abuso y el sexismo (Blaya, Debarbieux, y Lucas Molina, 2007). De la importancia de todo ello se hacen eco algunos autores que proponen el bilingüismo sexual como un nuevo modelo de convivencia entre los sexos, partiendo de sus diferencias y su necesidad de encuentro (Sáez Sesma, 2005). Coincidimos con Melendro, Suárez y Perdomo (2000) quienes defienden que hay que reinventar la educación en un sentido más amplio que el término, como proceso o conjunto de acciones por las que se forma para la vida desde la propia vida. Desde esta perspectiva, la educación tiene que ampliar los temas, las formas y los métodos para acceder a la cultura no sólo del saber académico sino también del saber vital, pretendiendo buscar un equilibrio armónico entre la formación racional y la liberación de la sensibilidad. En este contexto, las finalidades de la educación concuerdan con el desarrollo de una forma integral, inmersa en una cultura y una sociedad global (Santos Guerra et al., 2000).

En definitiva, como hemos expuesto de forma somera, una educación afectivo sexual concebida desde una perspectiva amplia debe abordar temas que van más allá de las relaciones sexuales. Debe también incluir formación y entrenar en competencias que conformen actitudes positivas hacia el bienestar sexual propio y ajeno y que contribuyan a conformar una mejor sociedad. Esta formación debe ir precedida de una evaluación de los conocimientos, competencias y actitudes de los participantes. Si bien existen estudios centrados en la evaluación de diferentes componentes afectivos-sexuales y que han utilizado un gran número de escalas (p.e. Diéguez, Sueiro, y López, 2003; Lameiras Fernández, Rodríguez, y Carrera, 2005; Ortega, Ojeda, Sutil, y Sierra Freire, 2005), con el presente trabajo queremos aportar información sobre las visiones de adolescentes hacia temas diversos pero englobados dentro de una concepción amplia de la educación afectivo-sexual. Empleamos para ello una breve escala más próxima a un formato abierto, que a modo de screening, permite identificar los conocimientos, conductas y actitudes hacia diferentes aspectos relacionados con la sexualidad y ayuda a fomentar el debate sobre las opiniones vertidas. Más concretamente, a través del presente estudio pretendemos: (1) conocer las actitudes, conocimientos y comportamientos de los adolescentes respecto a la sexualidad y sus prácticas, (2) Analizar posibles diferencias en función de variables como el género, el tipo de estudios, la procedencia rural o urbana o la concepción de la sexualidad.

Merece la pena en este punto precisar que en el presente estudio empleamos el término género como categoría teórica de análisis. Coincidimos con Pujal y Amigot (2010) quienes señalan que si: a) precisamos en cada análisis el uso teórico específico que hacemos de la categoría género, b) partimos de la inadecuación feminista de un uso meramente descriptivo, positivista, y psicologista y 
c) conectamos dicha categoría relacional con la dimensión polimorfa de las relaciones de poder en la sociedad contemporánea, dicha categoría teórica sigue siendo extremadamente útil y necesaria, y puede ser tomada como perspectiva para un análisis crítico.

Dicho esto, en el presente estudio esperamos encontrar: (1) asociaciones significativas entre el género y actitudes hacia la sexualidad y temas afines; (1) diferencias en la visión y prácticas de la sexualidad en función del género y de la concepción de la sexualidad; (2) ausencia de diferencias en función de los estudios cursados y la procedencia rural o urbana.

\section{Metodología}

\subsection{Participantes}

Los participantes consisten en una muestra de conveniencia de 141 estudiantes procedentes de un centro educativo concertado de la ciudad de Salamanca. De ellos, más de la mitad, el $58,9 \% \%$, son hombres y el $41,1 \%$ mujeres. En lo referente a la edad, el 58,9\% tienen entre 16 y 17 años , el 25,5\% tienen 18 años, y el 15,6\% tienen 19 o más años. El 22\% de los participantes son de pocedencia rural mientras que el $78 \%$ son de procedencia urbana. En cuanto a la formación, el 77,3\% cursan estudios de bachillerato y el 22,7\% realizan estudios de formación profesional de grado medio. El análisis de la posible asociación entre las variables sociodemográficas previamente señaladas puso de manifiesto la ausencia de asociación significativa entre la edad y el género (chi cuadrado $=0,228 ; \mathrm{gl}=2 ; \mathrm{p}=0,892$ ), entre formación y género (chi cuadrado $=1,670 ; \mathrm{gl}=1 ; \mathrm{p}=0,196)$, entre procedencia y género (chi cuadrado $=0,524$; $\mathrm{gl}=1 ; \mathrm{p}=0,469$ ) o entre procedencia y estudios (chi cuadrado=3,704; $\mathrm{gl}=1 ; \mathrm{p}=0,054$ ).Por otro lado y como era de esperar, se encontró una asociación significativa entre edad y estudios (Chi cuadrado $=60,230 ; \mathrm{gl}=2 ; \mathrm{p}=0,000)$ y los residuos tipificados indicaron que mientras que en bachillerato se acumula la población más joven, en los módulos profesionales se acumulan los participantes de mayor edad. También se encontró una asociación entre edad y procedencia (Chi cuadrad $=12,810 ; \mathrm{gl}=2 ; \mathrm{p}=0,002$ ) y el análisis de los residuos corregidos puso de manifiesto que es mucho más probable que el grupo de mayor edad proceda del ámbito rural. Así pues y con la salvedad que acabamos de comentar, es posible afirmar que los participantes se distribuyen de un modo bastante homogéneo en las diferentes variables sociodemográficas analizadas, pese a tratarse de una muestra de conveniencia y en la que por tanto, estas variables no se han podido controlar.

\subsection{Instrumentos}

El instrumento, denominado Cuestionario para adolescentes sobre educación sexual (CADES) consta de 30 items divididos en dos partes. En una primera se recogen datos generales 
sociodemográficos referidos al género, edad, procedencia estudios, etc. En una segunda parte se recogen datos de conocimientos sobre la sexualidad, hábitos y experiencias sexuales, actitudes hacia cuestiones sexuales o relacionadas con la sexualidad, que además tienen un matiz social como es el caso de la violencia de género o la prostitución. Su formato de respuesta es más abierto que los tradicionales cuestionarios de respuesta tipo Likert, pues las diferentes opciones de respuesta están pensadas para suscitar el intercambio de opiniones. Así, a diferencia de los instrumentos existentes, hemos tratado de desarrollar un cuestionario con preguntas de temas muy diversos, que sean susceptibles de promover la reflexión, discusión y debate.

Más concretamente, el apartado de Conocimientos incluye 12 items que aluden a métodos de prevención de enfermedades de transmisión sexual, anticonceptivos, etc. Se corrige otorgando un punto a las respuestas correctas y 0 puntos a las respuestas incorrectas y computando entonces el total de aciertos en cada caso. En segundo lugar y en lo que se refiere al apartado de apartado de hábitos y experiencia sexual, está compuesto por seis ítems y a su vez dividido en dos factores, por un lado el referido a la actividad sexual o experiencia en este campo, y que está compuesto de cuatro ítems, valorados en una escala de 1 a 4 puntos, indicando una mayor puntuación, una mayor experiencia sexual. Por otro lado, dos ítems miden el uso del preservativo y se valoran también de 1 a 4 puntos, indicando una mayor puntuación, más conductas de riesgo (véase en Anexo ítems 1 al 6).

En tercer lugar, la escala incluye 12 items sobre actitudes que se divide a su vez en cuatro factores: (1) 'visión de la sexualidad', compuesto por tres ítems $\left(\mathrm{n}^{\circ} .8,11\right.$ y 18) que ofrecieron una consistencia interna un Alfa de Cronbach=0,72; (2) 'visión de la homosexualidad, compuesto por cinco ítems ( $\mathrm{n}^{\circ}$. 7, 12, 13, 14 y 15) con una fiabilidad Alfa de Cronbach=0,68; (3) 'visión de la interrupción voluntaria del embarazo', compuesto por dos ítems ( $\mathrm{n}^{\mathrm{o}} .16$ y 17) que mostraron un Alfa de Cronbach=0,70; (4) 'visión de la prostitución', compuesto por dos ítems ( ${ }^{\circ} .9$ y 10) con una fiabilidad Alfa de Cronbach $=0,52$. Tras calcular las puntuaciones promedio de cada factor, hemos calculado las correlaciones entre los factores obteniendo una asociación significativa y positiva entre el factor visión de la sexualidad y el relativo a visión de la prostitución ( $\mathrm{rxy}=0,412 ; \mathrm{p}=0,001)$ (véanse en Anexo ítems 7 al 18).

\subsection{Procedimiento}

Los datos fueron recogidos durante el periodo de mayo y junio de 2013, en un centro concertado de Salamanca. Ello requirió la obtención de permisos por parte de la dirección del centro, así como la colaboración de orientadores y resto del profesorado. Los cuestionarios fueron rellenados aprovechando las horas de tutorías con los estudiantes, en horas de clase. La cumplimentación de los mismos se llevó a cabo en unos 20 minutos. La sencillez de las cuestiones, su formulación y el nivel educativo al que iban destinadas, hizo innecesario la resolución de dudas. Los estudiantes colaboraron voluntariamente tras garantizársele 
(verbalmente y por escrito) la total confidencialidad así como el anonimato de la información recogida. En informe final entregado al centro ofreció datos agrupados que garantizaran el cumplimiento de dichos compromisos éticos.

Para determinar la posible asociación entre variables sociodemográficas de los participantes, hemos empleado el estadístico Chi cuadrado. En segundo lugar, hemos utilizando estadísticos de los tests para determinar la fiabilidad y la validez del instrumento, concretamente hemos utilizado los estadísticos alfa de Cronbach para determinar la consistencia interna, y utilizado la correlación de Pearson para analizar las asociaciones entre las dimensiones de la escala. En tercer lugar, para el contraste de hipótesis hemos utilizado análisis Chi cuadrado en los casos oportunos, así como el procedimiento MLG multivariante para contrastar posibles diferencias entre grupos, controlando el hecho de que algunas variables correlacionan entre sí. Si los análisis multivariados resultan significativos, hemos empleado análisis de varianza para determinar en qué variables existen diferencias significativas. El nivel alfa establecido de antemano para los diferentes análisis fue de $\alpha=0,05$.

\section{Resultados}

Comenzamos dando respuesta a nuestro primer objetivo. En cuanto a las concepciones sobre la sexualidad, para un $73 \%$ de los participantes es algo que 'se tiene a lo largo de toda la vida'; un $26,2 \%$ considera que aparece en la adolescencia y un $0,7 \%$ manifiesta que desaparece en la vejez. Además, si bien un $79,4 \%$ considera que la sexualidad se refiere a aspectos sexuales, emocionales, afectivos, un 12,1\% indica que se refiere sólo a las conductas sexuales con otras personas, un $5,7 \%$ considera que se refiere sólo al sexo con o sin penetración y un $2,8 \%$ indica que se refiere sólo al sexo con penetración. En la Tabla 1 se incluyen las respuestas ofrecidas a los diferentes ítems que evalúan el grado de acuerdo con diferentes afirmaciones acerca de la sexualidad.

Tabla 1. Frecuencia y porcentaje de respuestas a los ítems sobre conocimientos

\begin{tabular}{|l|c|c|c|c|c|}
\hline & TA & BA & PM & BD & TD \\
\hline $\begin{array}{l}\text { La primera vez que una chica } \\
\text { hace el amor no puede quedarse } \\
\text { embarazada }\end{array}$ & $\begin{array}{c}4 \\
(2,84 \%)\end{array}$ & $\begin{array}{c}3 \\
(2,13 \%)\end{array}$ & $\begin{array}{c}26 \\
(18,44 \%)\end{array}$ & $\begin{array}{c}22 \\
(15,60 \%)\end{array}$ & $\begin{array}{c}86 \\
(60,99 \%)\end{array}$ \\
\hline $\begin{array}{l}\text { La mejor forma de evitar las } \\
\text { enfermedades de transmisión } \\
\text { sexual es usar preservativo en } \\
\text { toda relación sexual }\end{array}$ & $\begin{array}{c}116 \\
(82,27 \%)\end{array}$ & $\begin{array}{c}24 \\
(17,02 \%)\end{array}$ & $\begin{array}{c}1 \\
(0,71 \%)\end{array}$ & & \\
\hline
\end{tabular}




\begin{tabular}{|c|c|c|c|c|c|}
\hline $\begin{array}{l}\text { La píldora anticonceptiva y } \\
\text { otros métodos de estrógenos } \\
\text { sirven para prevenir las } \\
\text { enfermedades de transmisión } \\
\text { sexual }\end{array}$ & $\begin{array}{c}7 \\
(4,96 \%)\end{array}$ & $\begin{array}{c}11 \\
(7,80 \%)\end{array}$ & $\begin{array}{c}13 \\
(9,22 \%)\end{array}$ & $\begin{array}{c}32 \\
(22,70 \%)\end{array}$ & $\begin{array}{c}78 \\
(55,32 \%)\end{array}$ \\
\hline $\begin{array}{l}\text { El preservativo es seguro si se } \\
\text { coloca justo antes de la } \\
\text { eyaculación }\end{array}$ & $\begin{array}{c}8 \\
(5,67 \%)\end{array}$ & $\begin{array}{c}11 \\
(7,80 \%)\end{array}$ & $\begin{array}{c}26 \\
(18,44 \%)\end{array}$ & $\begin{array}{c}95 \\
(67,38 \%)\end{array}$ & $\begin{array}{c}1 \\
(0,71 \%)\end{array}$ \\
\hline $\begin{array}{l}\text { Hacer el amor en algunas } \\
\text { posturas evita el embarazo }\end{array}$ & $\begin{array}{c}1 \\
(0,71 \%)\end{array}$ & $\begin{array}{c}2 \\
(1,42 \%)\end{array}$ & $\begin{array}{c}19 \\
(13,48 \%)\end{array}$ & $\begin{array}{c}23 \\
(16,31 \%)\end{array}$ & $\begin{array}{c}96 \\
(68,09 \%)\end{array}$ \\
\hline $\begin{array}{l}\text { Con el preservativo se tiene } \\
\text { menos sensibilidad }\end{array}$ & $\begin{array}{c}36 \\
(25,53 \%)\end{array}$ & $\begin{array}{c}45 \\
(31,91 \%)\end{array}$ & $\begin{array}{c}38 \\
(26,95 \%)\end{array}$ & $\begin{array}{c}17 \\
(12,06 \%)\end{array}$ & $\begin{array}{c}5 \\
(3,55 \%)\end{array}$ \\
\hline $\begin{array}{l}\text { Los besos, las caricias y la } \\
\text { masturbación no son prácticas } \\
\text { sexuales porque no hay } \\
\text { penetración }\end{array}$ & $\begin{array}{c}3 \\
(2,13 \%)\end{array}$ & $\begin{array}{c}8 \\
(5,67 \%)\end{array}$ & $\begin{array}{c}20 \\
(14,18 \%)\end{array}$ & $\begin{array}{c}48 \\
(34,04 \%)\end{array}$ & $\begin{array}{c}62 \\
(43,97 \%)\end{array}$ \\
\hline $\begin{array}{l}\text { Las enfermedades } \\
\text { transmisión sexual sólo se } \\
\text { transmiten mediante el coito } \\
\text { vaginal }\end{array}$ & $\begin{array}{c}15 \\
(10,64 \%)\end{array}$ & $\begin{array}{c}17 \\
(12,06 \%)\end{array}$ & $\begin{array}{c}22 \\
(15,60 \%)\end{array}$ & $\begin{array}{c}33 \\
(23,40 \%)\end{array}$ & $\begin{array}{c}54 \\
(38,30 \%)\end{array}$ \\
\hline $\begin{array}{l}\text { La píldora del día después es un } \\
\text { método anticonceptivo }\end{array}$ & $\begin{array}{c}24 \\
(17,02 \%)\end{array}$ & $\begin{array}{c}27 \\
(19,15 \%)\end{array}$ & $\begin{array}{c}21 \\
(14,89 \%)\end{array}$ & $\begin{array}{c}28 \\
(19,86 \%)\end{array}$ & $\begin{array}{c}41 \\
(29,08 \%)\end{array}$ \\
\hline 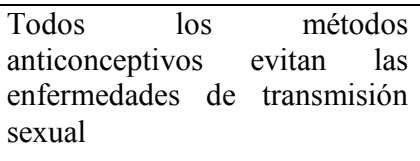 & $\begin{array}{c}8 \\
(5,67 \%)\end{array}$ & $\begin{array}{c}20 \\
(14,18 \%)\end{array}$ & $\begin{array}{c}21 \\
(14,89 \%)\end{array}$ & $\begin{array}{c}34 \\
(24,11 \%)\end{array}$ & $\begin{array}{c}58 \\
(41,13 \%)\end{array}$ \\
\hline $\begin{array}{l}\text { Las chicas, cuando están con el } \\
\text { periodo, no se pueden quedar } \\
\text { embarazadas }\end{array}$ & $\begin{array}{c}17 \\
(12,06 \%)\end{array}$ & $\begin{array}{c}11 \\
(7,80 \%)\end{array}$ & $\begin{array}{c}32 \\
(22,70 \%)\end{array}$ & $\begin{array}{c}26 \\
(18,44 \%)\end{array}$ & $\begin{array}{c}55 \\
(39,01 \%)\end{array}$ \\
\hline $\begin{array}{l}\text { La masturbación } \\
\text { efectos secundarios } \\
\text { como la } \\
\text { aparición de acné }\end{array}$ & $\begin{array}{c}5 \\
(3,55 \%)\end{array}$ & $\begin{array}{c}33 \\
(23,40 \%)\end{array}$ & $\begin{array}{c}42 \\
(29,79 \%)\end{array}$ & $\begin{array}{c}18 \\
(12,77 \%)\end{array}$ & $\begin{array}{c}43 \\
(30,50 \%)\end{array}$ \\
\hline
\end{tabular}

Fuente: Elaboración propia a partir de los análisis realizados con el SPSS v.15.

En cuanto a los comportamientos sexuales y ante la pregunta de qué hacen si alguien les atrae sexualmente, un 5\% indica que no hacen nada; un $67,9 \%$ manifiesta ir poco a poco y establecer una amistad con esa persona; un 9,3\% indica ser directo y e intentar convencer a esa persona para mantener relaciones, y un $17 \%$ manifiesta hacer lo que haga falta para tener sexo con esa persona. Ante la pregunta de si se han mantenido relaciones sexuales con otras personas, un $11,3 \%$ responde negativamente, un $26,2 \%$ manifiesta haber tenido sólo besos y caricias; un 10,6\% indica haber tenido relaciones sin penetración (petting) y un 51,8 \% indica haber tenido relaciones coitales. En cuanto al número de parejas sexuales que se han tenido, un $24,8 \%$ no ha tenido pareja alguna, un $27,7 \%$ ha tenido una pareja, un $22,0 \%$ ha tenido entre dos y tres personas diferentes y un $25,5 \%$ indica haber tenido más de tres parejas diferentes. 
Respecto a las personas con quienes se han mantenido relaciones sexuales, los participantes indican en un $45,6 \%$ que eran sus parejas, en un $35,1 \%$ eran amigos/as, en un $13,2 \%$ de los casos eran conocidos/as y en un $6,1 \%$ eran desconocidos/as. Por lo que respecta al uso del preservativo, un $60,5 \%$ manifiesta utilizarlo siempre, un $7,9 \%$ con personas que no son sus parejas, un $21,9 \%$ sólo cuando hay penetración y un $9,6 \%$ indican no utilizarlo nunca. Finalmente, cuando se les pregunta sobre las conductas que llevan a cabo cuando quieren tener sexo pero no tienen preservativo, un $31,0 \%$ manifiesta no hacer nada, pues sólo tienen relaciones con preservativo; un 19,5\% manifiesta practicar la "marcha atrás", un 45,1\% indica practicar petting y un 4,4\% indica practicar sexo sin protección.

Por lo que se refiere a las actitudes, del análisis de los ítems referidos a la visión de la homosexualidad se desprende lo siguiente. Un 90,1\% de los participantes consideran que las mujeres tienen el mismo derecho de los hombres de vivir su sexualidad libremente; un 9,2\% considera que si bien las mujeres tienen el mismo derecho que los hombres de vivir su sexualidad, deben ser más recatadas y sumisas que los hombres, y un $0,7 \%$ indican que las mujeres deben dejar las decisiones sobre su sexualidad en manos de los hombres. Sobre la consideración de la homosexualidad, un 9,9\% considera que es una enfermedad o un trastorno mental que debe ser tratado, un 20,6\% con consideran antinatural, un 37,6\% manifiestan que es una orientación sexual como cualquier otra y un 31,9\% consideran que cada uno es libre para estar con quien quiera. Además, los resultados indicaron que era más probable que los hombres consideraran que es antinatural (Chi cuadrado $=9,013 ; \mathrm{gl}=3 ; \mathrm{p}=0,029)$. Ante la pregunta de cuál es su actitud ante la homosexualidad, un 5,7\% indica que es mala, que lo considera aberrante, lo critica y persigue; un $32,6 \%$ indica que lo respeta pero no les parece bien; y un $61,7 \%$ indica tener buenas actitudes, de respeto y defensa. Continuando con este bloque de preguntas, ante la cuestión de cuál es la actitud cuando ven a dos chicas besándose o agarradas de la mano, un $13,5 \%$ manifiesta que lo rechazan o no les gusta verlas, un 3,5\% indican que les sorprende, un $14,2 \%$ indica que les gusta ver esas muestras de amor entre dos chicas y un $68,8 \%$ manifiestan que les da igual, pues lo que hagan los demás no es asunto suyo. Los análisis revelaron además que era más probable que a los hombres les guste ver esas muestras de amor entre mujeres, mientras que es más probable que a las mujeres les dé igual pues consideren que no es un asunto de su incumbencia (Chi cuadrado $=17,814 ; \mathrm{gl}=4 ; \mathrm{p}=0,001$ ). Ante la misma pregunta pero en este caso ante la homosexualidad masculina, un 43,3\% indican que lo rechazan o no les gusta verles; un 3,5\% indica que les sorprende verlo, un 4,3\% indica que les gusta ver esas muestras de amor entre dos chicos y un 48,9\% manifiestan que les da igual, pues lo que hagan los demás no es asunto. Es además bastante más probable que a los hombres les dé asco, mientras que para las mujeres sea un tema que no sea de su incumbencia (Chi cuadrado= 22,945; gl=4; $\mathrm{p}=0,000$ ).En la Figura 1 se exponen los resultados relativos a estas dos últimas cuestiones.

En cuanto a los principales resultados obtenidos en el factor referido a la visión de la sexualidad, un $78 \%$ consideraba que la sexualidad hay que vivirla y disfrutarla con responsabilidad, frente a un $6,4 \%$ que manifiesta que sólo debe ejercerse dentro del matrimonio, 
un $12,8 \%$ que indica que sólo debe practicarse con un fin reproductivo y un $2,8 \%$ que indica que no hay que ejercerla ya que es algo vergonzoso. Sobre la libertad sexual, para un $75,9 \%$ constituye un derecho para las mujeres y los hombres; un 3,5\% indican que es un derecho que tienen los hombres, un $12,1 \%$ manifiestan que es una aberración y un $8,5 \%$ consideran que es un pecado. Por lo que se refiere al sexo sin amor, un 40,4\% indica que está bien, pues amor y sexo son dos cosas diferentes; un $41,1 \%$ indica que lo respetan pero nunca lo practicarían; un $14,9 \%$ lo considera inconcebible y un 3,5\% indica que les da igual que sea con o sin amor siempre que se haga dentro del matrimonio y con fines reproductivos. Se aprecia además que es bastante más probable que los hombre consideren que sexo y amor son dos cosas distintas (Chi cuadrado=10,610; $\mathrm{gl}=3 ; \mathrm{p}=0,014)$.

\section{Figura 1. Distribución de respuestas sobre actitudes hacia la homosexualidad femenina y masculina, en función del género de los respondientes}
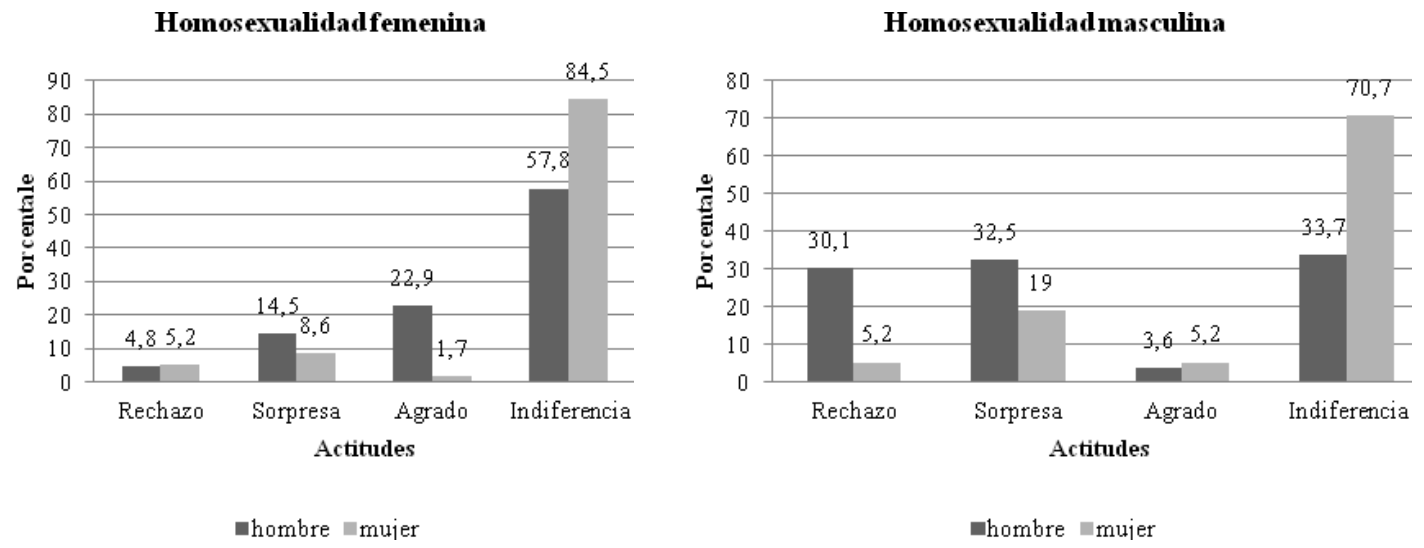

Fuente: Elaboración propia a partir de los análisis realizados con el SPSS v.15.0

Centrándonos en el tema de la prostitución, un 16,3\% lo ve como un trabajo más, que no tiene nada de malo; un $11,3 \%$ lo considera una actividad necesaria para la sociedad; un 52,5\% lo considera una actividad que las personas mayoritariamente mujeres realizan por necesidad y un $19,9 \%$ lo consideran una actividad denigrante para la mujer que debería estar perseguida. En cuando a lo que sienten cuando ven a una prostituta, un $22,7 \%$ manifiesta sentir asco y vergüenza; un $31,9 \%$ indica experimentar pena y lástima; un 20,6\% siente empatía y un $24,8 \%$ indica sentir indiferencia. Además, es más probable que las mujeres indiquen sentir pena y lástima que los varones (Chi cuadrado $=25,747 ; \mathrm{gl}=3 ; \mathrm{p}=0,000$ ).

Por último y respecto a la visión de la interrupción voluntaria del embarazo, un 48,9\% indica que les parece bien, pues cada uno decide sobre su vida; un 38,3\% indican que les parece bien solo en algunos casos (violaciones, salud de la madre o salud del feto); un 6,4\% indica que 
lo respetan pero nunca lo harían y un $6,4 \%$ indica que no se debe hacer bajo ningún concepto, pues atenta contra el derecho a la vida. Ante la pregunta de ¿Cómo crees que actuarías si descubres que una amiga tuya ha abortado voluntariamente?, un $65, \%$ indica que la apoyaría y restarían importancia al asunto, pues ha hecho lo mejor para todos; un 24,3\% indica que la apoyaría y le dejarían claro que lo que ha hecho no está bien; un 9,3\% manifiesta que la respetaría pero no la trataría igual, pues le ha decepcionado, y un 1,4\% indica que se alejarían de ella, pues no la podrán perdonar nunca.

Ofrecemos a continuación las puntuaciones promedio y dispersión, obtenidas en las diferentes variables por los participantes analizados (véase Tabla 2). Se puede apreciar cómo el nivel de conocimientos podría calificarse como medio, con una importante dispersión. En cuanto a los factores actitudinales, las puntuaciones promedio más elevadas se obtienen los factores referidos a la visión de la sexualidad y de la interrupción voluntaria del embarazo, mientras que las más bajas se obtienen en el factor 4 referido a la prostitución. Respecto a diferencias entre géneros, se observa cómo salvo en el factor 3 de visión de la homosexualidad, en todos los demás factores los hombres puntúan más elevado que las mujeres.

Pasamos a exponer los resultados relativos al contraste de nuestra segunda hipótesis. Así y en relación a las posibles diferencias en función del género, los análisis multivariados reflejaron la existencia de diferencias significativas [Lamba de Wilks $=0,710 ; \mathrm{F}_{(7,106)}=6,198 ; \mathrm{p}=0,001$; $\left.\eta^{2}=0,290\right]$. Los análisis bivariados reflejaron diferencias significativas en función del género en el factor 2 de visión de la homosexualidad $\left[\mathrm{F}_{(1,112)}=7,256 ; \mathrm{p}=0,000 ; \eta^{2}=0,155\right]$, en el factor 4 sobre la visión de la prostitución $\left[\mathrm{F}_{(1,112)}=4,475 ; \mathrm{p}=0,012 ; \eta^{2}=0,055\right]$ y en el factor referido a la actividad sexual $\left[F_{(1,112)}=1,810 ; p=0,014 ; \eta^{2}=0,052\right]$. Las mujeres puntuaron significativamente más elevado que los hombres en lo referido a la homosexualidad y actividad sexual, aunque más bajo que los hombres en la visión de la prostitución.

Tabla 2. Estadísticos descriptivos de las variables de interés

\begin{tabular}{llll}
\hline Variables & $\begin{array}{l}\text { Total (N=141) } \\
\text { Media (DT) }\end{array}$ & $\begin{array}{l}\text { Hombres (N=83) } \\
\text { Media (DT) }\end{array}$ & $\begin{array}{l}\text { Mujeres (N=58) } \\
\text { Media (DT) }\end{array}$ \\
\hline $\begin{array}{l}\text { Conocimientos } \\
\text { Actitudes: }\end{array}$ & $5,51(2,53)$ & $5,65(2,47)$ & $5,31(2,62)$ \\
F1. Visión de la sexualidad & $3,42(0,73)$ & $3,43(0,74)$ & $3,39(0,71)$ \\
F2. Visión de la homosexualidad & $3,29(0,63)$ & $3,11(0,62)$ & $3,55(0,54)$ \\
F3. Visión del aborto & $3,41(0,68)$ & $3,52(0,62)$ & $3,26(0,74)$ \\
F4. Visión de la prostitución & $2,36(0,85)$ & $2,53(0,88)$ & $2,11(0,73)$ \\
Actividad sexual & $2,41(0,69)$ & $2,57(0,66)$ & $2,19(0,68)$ \\
Uso del preservativo & $4,02(1,62)$ & $4,14(1,66)$ & $3,81(1,57)$ \\
\hline
\end{tabular}

Fuente: Elaboración propia a partir de los análisis realizados con el SPSS v.15.0 
En segundo lugar hemos analizado las posibles diferencias en función de la concepción del sexo como algo positivo o bueno sin restricción alguna o con ciertas restricciones. Los análisis multivariados reflejaron la existencia de diferencias significativas [Lamba de Wilks $=0,880 ; \mathrm{F}_{(7}$, 106) $\left.=2,066 ; p=0,05 ; \eta^{2}=0,120\right]$. Los análisis bivariados reflejaron diferencias significativas en función de la concepción de la sexualidad en el factor 3 visión de la interrupción del embarazo $\left[\mathrm{F}_{(1,112)}=1,408 ; \mathrm{p}=0,038 ; \eta^{2}=0,038\right]$ y en el factor referido a la actividad sexual $\left[\mathrm{F}_{(1,112)}=5,040\right.$; $\left.\mathrm{p}=0,027 ; \eta^{2}=0,043\right]$. En ambos casos, quienes consideran que el sexo es algo natural, bueno y positivo, puntúan significativamente más alto en ambos factores que quienes consideran que el sexo es algo natural, bueno y positivo pero tiene ciertas restricciones.

Por lo que se refiere al contraste de nuestra tercera hipótesis, y comenzando por el análisis del posible impacto del tipo de estudios cursados, los análisis multivariados reflejaron la existencia de diferencias significativas [Lamba de Wilks $=0,212 ; \mathrm{F}_{(7,106)}=56,432 ; \mathrm{p}=0,000$; $\left.\eta^{2}=0,788\right]$. Los análisis bivariados reflejaron diferencias significativas en función de los estudios que se cursan en el factor 1 de visión de la sexualidad $\left[\mathrm{F}_{(1,112)}=45,341 ; \mathrm{p}=0,000\right.$; $\left.\eta^{2}=0,733\right]$, en el factor referido a la visión de la prostitución $\left[F_{(1,112)}=24,891 ; p=0,000\right.$; $\left.\eta^{2}=0,308\right]$, y en el referido a la actividad sexual $\left[\mathrm{F}_{(1,112)}=10,417 ; \mathrm{p}=0,002 ; \eta^{2}=0,085\right]$. Los análisis indicaron que si bien en el factor de actitudes hacia la sexualidad y prostitución, los estudiantes de bachillerato obtuvieron puntuaciones significativamente superiores $(M=3,34$; $\mathrm{DT}=0,38)$ a los estudiantes de módulos profesionales $(\mathrm{M}=2,02 ; \mathrm{DT}=0,39)$, en el caso de la actividad sexual los estudiantes de bachillerato obtuvieron puntuaciones significativamente inferiores $(M=2,54 ; D T=0,51)$ que los estudiantes de módulos profesionales $\quad(M=2,92$; $\mathrm{DT}=0,61$ ). Por otro lado, el factor procedencia (rural o urbana) no arrojó diferencias significativas en los contrastes multivariados [Lamba de Wilks $=0,914 ; F_{(7,106)}=1,452 ; p=0,20$; $\left.\eta^{2}=0,086\right]$.

\section{Conclusiones}

El presente estudio pretende contribuir a incrementar el debate en las aulas sobre temas relacionados con la educación afectivo sexual desde una perspectiva amplia, en la que se incluyan temas más allá de lo meramente biológico, médico, y se indica en aspectos más sociales, culturales, relacionados con el género, los estereotipos, la violencia y otros aspectos relacionados. Varios resultados merecen la pena ser destacados.

En primer lugar y en cuanto a los conocimientos mostrados por los alumnos de estudios post-obligatorios (bachiller y módulos profesionales), los resultados indican que es necesario incrementar sustancialmente la información que éstos tienen sobre temas sexuales, pues la tasa de errores o de respuestas que indican un desconocimientos de temas básicos como la prevención de los embarazos o de las ETS, o de los efectos de la masturbación, es alarmante. 
Por ello y pese a que en este trabajo incidimos en aspectos más socioculturales, no debemos perder de vista la necesidad de educar en aspectos básicos como los expuestos, en la línea de lo que vienen realizando otros autores (Bausela Herreras, 2005; Carrera-Fernández et al., 2007; Claramunt Busó, Hernansaiz Cañete, y León Zarceño, 2004; Espada Sánchez y Quiles Sebastián, 2002; Gil García, González López, y Meneses Falcón, 2010; Lameiras Fernández, Rodríguez, y Carrera, 2005; Ubillos Landa y Mayordomo López, 2003).

En segundo lugar y en cuanto a los comportamientos sexuales de los participantes, pese a tratarse de una evaluación realizada mediante cuestionarios y que por tanto, tan sólo mide la intención conductual o la conducta informada y no la conducta en sí misma, se aprecian comportamientos que requieren una reflexión como son:

- El hecho de que un $17 \%$ indique hacer lo que sea por tener sexo con una persona que les resulte atractiva.

- La evidencia de que tan sólo un 60,5\% de los informantes manifiesta utilizar siempre el preservativo, lo que nos deja a un 30,5\% de los jóvenes de este estudio en situación clara de riesgo ante ETS y embarazos no deseados.

En tercer lugar y en contra de nuestras predicciones, hemos encontrado que el tipo de estudios cursados ejerce una influencia en los participantes, al menos en cuanto a su consideración de la sexualidad, la prostitución o la actividad sexual. Si bien, posibles factores como la edad o la procedencia pudieran estar incidiendo en estos resultados, posteriores estudios con muestras más amplias y con procedimientos de selección más controlados contribuirán a arrojar más luz sobre este tema.

En cuarto y último lugar, a lo largo de todo el estudio se pone de manifiesto un claro efecto de la variable género, tanto en la concepción de la homosexualidad como de la prostitución y en la propia actividad sexual. Destacamos entre otros, los siguientes resultados obtenidos:

- Presencia de actitudes sexistas ante el modo en que la mujer debe vivir la sexualidad.

- Persistencia de una diferenciación tradicional de géneros en lo relativo a la distinción entre sexo y amor por parte del hombre, frente a la vivencia del mismo por parte de la mujer.

- Actitudes homófobas, sobre todo por parte de la población masculina y ante la homosexualidad masculina, que pone á estos jóvenes homosexuales en una clara situación de riesgo de experimentar rechazo, cuando no otras conductas más agresivas, como también ha puesto de manifiesto la literatura, ante quienes no se ajustan a las normas de género (Platero Méndez, 2010).

- Actitudes más compasivas hacia la prostitución por parte de las mujeres, frente a una actitud más indiferentes por parte de los hombres, que sugiere de nuevo una clara incidencia de los roles de género.

Además, los resultados indican que la concepción de la sexualidad se encuentra asociada a su vez con actitudes hacia otros aspectos como la interrupción del embarazo o la actividad 
sexual. La relación entre actitudes y conducta y entre éstas y diferentes referentes actitudinales (prostitución, aborto, homosexualidad...) ha sido puesto de manifiesto en otros estudios (Blaya, Debarbieux, y Lucas Molina, 2007; Sueiro Domínguez, Diéguez, Chas, y Diz, 2004) y el presente trabajo no hace sino ofrecer apoyo adicional a la necesidad de llevar a cabo programas de educación afectivo sexual que incluyan una profunda reflexión sobre todos estos temas.

No queremos terminar sin antes aludir a varias limitaciones del presente estudio. En primer lugar, se trata de un estudio realizado en un único centro educativo de Salamanca. Posteriores estudios con un muestreo estratificado y con muestras más amplias permitirán contrastar los resultados preliminares aquí obtenidos. En segundo lugar, la información ha sido obtenida mediante cuestionarios y por tanto, es susceptible de experimentar fuentes de error procedentes de los participantes (deseabilidad social, ausencia de sinceridad), así como del propio instrumento de medida. Posteriores estudios con muestras más amplias y diversas permitirán la puesta a prueba de las propiedades psicométricas del instrumento, lo que requerirá probablemente la inclusión de un mayor número de ítems para ampliar la validez de contenido de varios de sus factores. En tercer lugar y como hemos indicado, el método de recogida de información podría complementarse con otras técnicas (p.e. grupos focales, observación), para así triangular la información recogida. Ello incrementará sin lugar a dudas la precisión de los datos obtenidos. Estas y otras limitaciones no hacen sino animarnos a seguir profundizando en este tema, en aras de la promoción en nuestros estudiantes de unos conocimientos, competencias, habilidades y valores que les permitan hacer elecciones que mejoren sus vidas y contribuyan al mismo tiempo a mejorar la sociedad.

\section{BIBLIOGRAFÍA}

- Abad, María Luisa (2002): Género y educación: la escuela coeducativa. Caracas, Venezuela: Editorial Laboratorio Educativo.

- Anabitarte Rivas, Héctor (2002): “VIH/SIDA: 20 años después, ¿qué hay que hacer?”. En: Revista Española de Drogodependencias, nº. 27, pp. 529-534.

- Bataller i Perelló, Vicente (2002): "Prevención del VIH/SIDA en la adolescencia y juventud en el marco integral de la educación para la salud". En: Revista Española de Drogodependencias, $\mathrm{n}^{\circ} .27$, pp. 428-437.

- Bausela Herreras, Esperanza (2005): "Diseño de un programa de prevención de embarazos no deseados centrado en el desarrollo de las habilidades sociales y de autoestima dirigido a alumnos de educación secundaria obligatoria”. En: Revista de Psicodidáctica, $\mathrm{n}^{\circ}$. 10 , pp.103-120. 
- Blaya, Catherine; Debarbieux, Eric y Lucas Molina, Beatriz (2007): "La violencia hacia las mujeres y hacia otras personas percibidas como distintas a la norma dominante: el caso de los centros educativos". En: Revista de Educación (Madrid) , nº. 342, pp.61-81.

- Caricote Agreda, Esther (2006): "Influencia de los estereotipos de género en la salud sexual en la adolescencia”. En: EDUCERE, n. 34, pp. 463 - 470.

- Carrera-Fernández, María Victoria et al. (2007): "Evaluación de un programa de educación sexual con estudiantes de Educación Secundaria Obligatoria". En: International Journal of Clinical and Health Psychology, $\mathrm{n}^{\circ} .7, \mathrm{pp} .739-751$.

- Carrillo, Isabel; Prat, Pilar; Guitart, Rosa y Fatsini, Esther (2010): "Investigar para desvelar los modelos de género en la adolescencia". En: Temps d'Educació, no . 39, pp.125138.

- Claramunt Busó, Consuelo; Hernansaiz Cañete, Beatriz y León Zarceño, Eva (2004): “La salud sexual en la educación: conocimientos y actitudes sexuales en la etapa adolescente". En: Informació Psicològica, $\mathrm{n}^{\mathrm{o}}$. 85/86, pp.50-59.

- Colectivo Harimaguada (2007): Sexpresan. Multimedia para la Educación AfectivoSexual Madrid: ITE, Ministerio de Educación.

- Corona, Esther (1994): "Identidades de género en busca de una teoría". En: Conapo: Antología de la sexualidad humana I. México, Conapo/Miguel Ángel Porrúa, pp. 299-314

- Costa, Malena (2006): “Distintas consideraciones sobre el binarismo sexo/género". En: $A$ Parte Rei. Revista de Filosofía, no ${ }^{\circ}$ 46, pp. 1-7.

- da Silva, Denise Quaresma (2013): "Lo legitimado y lo estigmatizado: Género y sexualidad en la educación sexual". En: Revista Interamericana de Psicología , nº 47, pp.441-448.

- Diéguez, José Luis; Sueiro, Encarnación y López., F. (2003): “The Sexual double standard y variables relacionadas". En: Cuadernos de Medicina Psicosomática y Psiquiatría de Enlace, $\mathrm{n}^{\circ} .67 / 68$, pp.79-88.

- Diz, M. C.; Sueiro, Encarnación; Chas, M. D. y Diéguez, José Luis (2003):. "Comportamientos sexuales de adolescentes del medio rural gallego ( $3^{\mathrm{a}}$ Parte) ". En: Cuadernos de Medicina Psicosomática y Psiquiatría de Enlace, no . 67/68, pp.46-60.

- Espada Sánchez, José Pedro y Quiles Sebastián, María José (2002): “Prevención del SIDA en la Escuela: pautas para la elaboración de un programa comportamental-educativo". En: Revista Española de Drogodependencias, no. 27, pp.381-396.

- García Martínez, Alfonso (2005): "Educación y prevención del SIDA". En: Anales de Psicología, n'. 21, pp.50-57.

- Gil García, Eugenia; González López, José Rafael y Meneses Falcón, Carmen (2010): "Percepción del riesgo y dinámicas promotoras de salud en adolescentes: una mirada de género". En: Revista Española de Drogodependencias, nº. 35, pp.297-308.

- González Gabaldón, Blanca (1999): "Los estereotipos como factor de socialización en el género". En: Comunicar, no. 12, pp. 79-88.

- González Pérez, Teresa (2010): "Mujeres, educación y democracia”. En: Revista de Educación (Madrid), no. 351, pp.337-359.

- González Pozuelo, Fernando (2008): "Estereotipos de género y actitudes sexistas de la población escolar extremeña”. En: Barataria. Revista castellano-manchega de Ciencias Sociales, $\mathrm{n}^{\circ} .9$, pp.37-61. 
- Hernando Gómez, Ángel (2007): "La prevención de la violencia de género en adolescentes: una experiencia en el ámbito educativo". En: Apuntes de Psicología, nº. 25, pp.325-340.

- Igartúa Perosanz, Juan José y Rodríguez Bravo, Ángel (2002): “Intervenciones mediáticas par la prevención del SIDA: la aproximación educación-entretenimiento". En: Revista Española de Drogodependencias, $\mathrm{n}^{\circ}$. 27, pp.397-406.

- Jara Rascón, José y Alonso Sandoica, Esmeralda (2008): "Ética y eficacia en las campañas de salud sexual". En: Cuadernos de Bioética, no. 19, pp.81-93.

- Lameiras Fernández, María; Rodríguez, Yolanda y Carrera, María Victoria (2005): "Evaluación cuantitativa de un programa de educación afectivo-sexual". En: Cuadernos de Medicina Psicosomática y Psiquiatría de Enlace, $\mathrm{n}^{\mathrm{0}}$. 73, pp.48-58.

- Lobato, Lidia y Jenaro, Cristina (2013): "La educación afectivo-sexual, ¿una vía para la igualdad? Caso de los jóvenes de la provincia de Salamanca". Universidad de Salamanca, Máster universitario en Antropología aplicada: salud y desarrollo comunitario, Salamanca.

- Melendro, Miguel; Suárez, Luis Ámgel y Perdomo, Silvia (2000): Adolescentes y jóvenes en dificultad social. Madrid: Cáritas Española Editores.

- Muñoz Cadavid, María A. y Revenga Sánchez, Margarita (2005): “Aprendizaje y educación afectivo - sexual: una revisión de los planteamientos iniciales del aprendizaje de las cuestiones sexuales". En: Revista Galego-Portuguesa de Psicoloxía e Educación, nº ${ }^{\circ}$ 12, pp.45-56.

- Ortega, Virgilio; Ojeda, Pilar; Sutil, Francisco y Sierra Freire, Juan Carlos (2005): "Culpabilidad sexual en adolescentes: estudio de algunos factores relacionados". En: Anales de Psicología, no. 21, pp.268-275.

- Ortiz, Luis (2004): “La opresión de minorías sexuales desde la inequidad de género". En: Política y Cultura, $\mathrm{n}^{\circ}$. 22, pp.161-182.

- Ortiz, Luis y Granados, José Arturo (2003): "Violencia hacia bisexuales, lesbianas y homosexuales de la ciudad de México". En: Revista Mexicana de Sociología, nº. 65, pp. 265-303.

- Palomino, Jesús (2003): “Un nuevo horizonte para el desarrollo de la educación sexual en el ámbito escolar". En: Estudios de Juventud, nº. 63, pp.75-80.

- Pérez Jiménez, Lourdes (2010): “Comunicación experiencia: Educación de los sexos. Prevención de la violencia en parejas jóvenes”. En: Anuario de Sexología, nº 12, pp.79-82.

- Planes Pedra, Montserrat et al. (2004): "Prevención de la transmisión sexual del VIH en adolescentes". En: Cuadernos de Medicina Psicosomática y Psiquiatría de Enlace, n . 71-72, pp.76-85.

- Platero Méndez, Raquel (2010): "Sobrevivir al Instituto y a la Facultad: voces y vivencias sobre la heteronormatividad, la homofobia y la masculinidad de las chicas". En: Revista de Estudios de Juventud, $\mathrm{n}^{\circ} .89$, pp.39-58.

- Pujal Llombart, Margot y Amigot Leache, Patricia (2010): "El binarismo de género como dispositivo de poder social, corporal y subjetivo". En: Quaderns de Psicologia, vol. 2, nº 12, pp.131-148.

- Sáez Sesma, Estefanía (2009): "Los profesionales de la sexología en la educación sexual". En: Anuario de Sexología, no. 11, pp.89-94.

- Sáez Sesma, Silberio (2005): "Reflexiones sobre la agresividad. Para controlar y prevenir la violencia". En: Anuario de Sexología, nº. 9, pp.85-100. 
- Samaniego García, Esperanza y Freixas Farré, Ana (2010): "Estudio sobre la identificación y vivencia de violencia en parejas adolescentes". En: Apuntes de Psicología, $\mathrm{n}^{\mathrm{o}} .28$, pp.349-366.

- Santos Guerra, Ángel M. et al. (2000): El harén pedagógico: perspectiva de género en la organización escolar. Barcelona: Grao.

- Sueiro Domínguez, Encarnación: Diéguez, J. L.; Chas, M. D. y Diz, M. C. (2004): "Primeras fuentes de información, conocimientos, actitudes y prácticas sexuales. Su interrelación”. En: Cuadernos de Medicina Psicosomática y Psiquiatría de Enlace, nº 69-70, pp.95-114.

- Ubillos Landa, Silvia y Mayordomo López, Sonia (2003): "La Prevención Sexual del VIH/SIDA en España". En: Revista Española de Drogodependencias, nº. 28, pp.328-343.

- Usieto, Ricardo y Sastre, Julia (2002): “La información y educación sobre el SIDA como estrategia de prevención en los jóvenes. Una experiencia concreta entre los adolescentes escolarizados de Cantabria". En: Revista Española de Drogodependencias, n ${ }^{\circ}$ 27, pp.445465.

- WHO Regional Office for Europe (2010): Estándares de Educación Sexual para Europa. Madrid: Madrid Salud. Servicio de Prevención y Promoción de la Salud y Salud Ambiental.

\section{ANEXO}

\section{CADES-Cuestionario para Adolescentes Sobre Educación Sexual (Lobato y Jenaro, 2013)}

(Nota: entre paréntesis y en subíndice se indican las claves de corrección)

\section{Si alguien te atrae sexualmente}

$\square$ No haces nada $_{(1)}$

Vas poco a poco y estableces una amistad con ella $(2)$

$\square$ Eres directo y la intentas convencer para mantener relaciones ${ }_{(3)}$

Haces lo que haga falta para tener sexo con ella $(4)$

\section{2. ¿Has mantenido relaciones sexuales con otras personas?}

No, ninguna ${ }_{(1)}$

Sólo besos y caricias ${ }_{(2)}$

Relaciones sin penetración (petting) $(3)$

Relaciones coitales ${ }_{(4)}$

3. Con cuántas personas has tenido relaciones sexuales

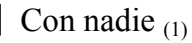

Con una persona $(2)$

$\square$ Con dos o tres personas diferentes $(3)$

Con más de tres personas diferentes ${ }_{(4)}$

4. La persona o las personas con las que has mantenido relaciones sexuales:

Eran tus parejas (1)

Eran amigos/as $(2)$ 
Eran conocidos/as $(3)$

Eran desconocidos/as $(4)$

\section{Utilizas preservativo:}

Siempre $_{(1)}$

Con personas que no son mis parejas $(2)$

Sólo cuando hay penetración ${ }_{(3)}$

Nunca $_{(4)}$

\section{Cuando quieres tener sexo pero no tienes preservativo:}

No haces nada, sólo tienes relaciones con preservativo $(1)$

$\square$ Practicas la "marcha atrás"

Practicas sexo sin penetración (petting) $(3)$

Te da igual, practicas sexo sin protección ${ }_{(4)}$

\section{Las mujeres:}

$\square$ Tienen el mismo derecho de los hombres de vivir su sexualidad libremente ${ }_{(4)}$

Tienen el mismo derecho que los hombres de vivir su sexualidad pero deben ser más recatadas y sumisas que ellos $_{(3)}$

Deben dejar las decisiones sobre su sexualidad en manos de los hombres ya que ellas no tienen personalidad para decidir $(2)$

Tienen que olvidar su sexualidad y adaptarse a los deseos de los hombres $(1)$

\section{La sexualidad:}

Hay que vivirla y disfrutarla con responsabilidad ${ }_{(4)}$

Sólo debe ejercerse dentro del matrimonio $(3)$

Sólo debe practicarse con un fin reproductivo, todo lo demás está mal (2)

No hay que ejercerla ya que es algo vergonzoso $(1)$

\section{Ves la prostitución:}

Como un trabajo más que no tiene nada de malo ${ }_{(4)}$

Como una actividad necesaria para la sociedad $(3)$

$\square$ Como una actividad que las personas mayoritariamente mujeres realizan por necesidad ${ }_{(2)}$

Como una actividad denigrante para la mujer que debería estar perseguida $(1)$

\section{Cuando ves una prostituta:}

Sientes asco y vergüenza $(1)$

Sientes pena y lástima $(2)$

Sientes empatía (3)

Te es indiferente ${ }_{(4)}$

\section{Opinas que la libertad sexual:}

$\square$ Es un derecho para las mujeres y los hombres ${ }_{(4)}$

$\square$ Es un derecho que tienen los hombres ${ }_{(3)}$

$\square$ Es una aberración $_{(2)}$

Es un pecado $_{(1)}$ 


\section{Crees que la homosexualidad:}

$\square$ Es una enfermedad o un trastorno mental que debería ser tratado $(1)$

$\square$ Es antinatural porque las mujeres tienen que estar con hombres y viceversa (2)

$\square$ Es una orientación sexual como cualquier otra $(3)$

Es algo positivo, que cada uno esté con quien quiera ${ }_{(4)}$

13. ¿Cuál es tu actitud ante la homosexualidad?

$\square$ Mala, crees que es aberrante ${ }_{(1)}$

$\square$ La respetas pero no te parece bien $(2)$

$\square$ No te gusta, la criticas y la persigues ${ }_{(3)}$

$\square$ Buena, la respetas y defiendes $(4)$

14. Cuando ves a dos chicas besándose o agarradas de la mano:

Te da asco verlas y le dices que no lo sigan haciendo (1)

No te gusta verlas, pones mala cara y miras para otro lado (2)

Te sorprende y avergüenza $(3)$

Te gusta ver esas muestras de amor entre dos chicas $(4)$

Te da igual, lo que hagan los demás no es asunto tuyo ${ }_{(5)}$

15. Cuando ves a dos chicos besándose o agarrados de la mano:

$\square$ Te da asco verlos y le dices que no lo sigan haciendo (1)

No te gusta verles, pones mala cara y miras para otro lado $(2)$

$\square$ Te sorprende y avergüenza $(3)$

$\square$ Te gusta ver esas muestras de amor entre dos chicos $(4)$

$\square$ Te da igual, lo que hagan los demás no es asunto tuyo ${ }_{(5)}$

\section{La interrupción voluntaria del embarazo:}

$\square$ Me parece bien, cada uno decide sobre su vida (4)

$\square$ Me parece bien solo en algunos casos (violaciones, salud de la madre o salud del feto) (3)

$\square$ Lo respetas pero nunca lo harías ${ }_{(2)}$

No se debe hacer bajo ningún concepto. Atenta contra el derecho a la vida (1)

17. ¿Cómo crees que actuarías si descubres que una amiga tuya ha abortado voluntariamente?

$\square$ La apoyarías y le restarías importancia al asunto. Ha hecho lo mejor para todos ${ }_{(4)}$

$\square$ La apoyarías y le dejarías claro que lo que ha hecho no está bien ${ }_{(3)}$

$\square$ La respetarías pero no la tratarías igual. Te ha decepcionado ${ }_{(2)}$

Te alejarías de ella, ha hecho mal y no la podrás perdonar nunca ${ }_{(1)}$

\section{El sexo sin amor:}

Está bien, el amor y el sexo son dos cosas diferentes ${ }_{(4)}$

Lo respetas pero nunca lo practicarías con alguien que no quieres $(3)$

Es inconcebible, el sexo es la expresión del amor y no debe hacerse si no lo hay (2)

Te da igual que sea con o sin amor siempre que se haga dentro del matrimonio y con fines reproductivos $_{(1)}$ 